\title{
Evaluación de un prototipo didáctico de Refrigeración Solar
}

\author{
Harenia del Carmen Maldonado Úbeda ${ }^{1}$ \\ María José Talavera Meneses ${ }^{2}$ \\ Edwin Antonio Reyes Aguilera ${ }^{3}$ \\ Luis Lorenzo Fuentes Peralta ${ }^{4}$
}

\section{RESUMEN}

El objetivo principal de esta investigación, es evaluar los parámetros característicos de funcionamiento de un prototipo de refrigeración solar. Es una investigación cuantitativa y cualitativa según su enfoque, con un nivel de profundidad según su tipología: descriptiva. La muestra del estudio es probabilístico. Los instrumentos utilizados fueron: la encuesta y una guía diseñada para recolección de datos, se instalaron manómetros de presión, termopares para medición de temperatura y solarimetro para el monitoreo de la radiación. Los resultados obtenidos muestran que las variables del refrigerador solar dependen completamente de la radiación solar, esto debido a que cuando esta era mayor, se logran alcanzar temperaturas entre los 4 oC y 6 oC, rango requerido para el mantenimiento y conservación de vacunas $\mathrm{u}$ otros tipos de medicamentos, utilizados en puestos de salud, según las respuesta de los estudiantes, encuestados de los diferentes años de la carrera de Ingeniería en Energías Renovables, el constar para fines didácticos con un prototipo de refrigeración solar es importante debido a que permite ampliar los conocimientos mediante la vinculación de la teoría recibida en aula de clase con la práctica. Se concluye que el aprovechamiento de la energía solar para el funcionamiento de un refrigerador solar, beneficia los estudiantes ya que pueden realizar prácticas enfocadas a estos temas.

Palabras claves: Refrigerador solar, Radiación, Presión, Temperatura.

Recibido: 09 de diciembre de 2015

Aceptado: 29 de enero de 2016

1 UNAN-Managua/FAREM-Estelí. Correo Electrónico: maldonadoubedaharenia@yahoo.com

2 UNAN-Managua/FAREM-Estelí. Correo Electrónico: marijoseth@yahoo.es

3 UNAN-Managua/FAREM-Estelí. Correo Electrónico: edwinra11@yahoo.es

4 UNAN-Managua/FAREM-Estelí. Correo Electrónico: 1lfuentesp072@yahoo.com.mx 


\section{Evaluation of an educational prototype of Solar Cooling}

\section{SUMARY}

The main objective of this research is to evaluate the characteristic parameters of the operating process of a solar cooling prototype. It is a quantitative qualitative and descriptive research. The study sample is probabilistic. The instruments used were: the survey and a designed guide to collect data, pressure gauges, and thermocouples for temperature measurement and a solarimeter for radiation monitoring were installed. The results show that the variables of solar refrigerator dependent completely on the solar radiation, this because when this was greater, they manage to reach temperatures between $4^{\circ} \mathrm{C}$ and $6^{\circ} \mathrm{C}$, required range for the maintenance and preservation of vaccines or other drugs used in health care centers, according to the of the students' responses who were surveyed in the different courses of the Renewable Energies Engineering, having a solar cooling prototype for didactic purposes, is important because it can increase the knowledge by linking the theory received in classroom with the practice. The use of solar energy for the operation of a solar refrigerator, benefits students because they can do internships focused on these issues.

Keywords: Solar Cooling, radiation, pressure, temperature . 


\section{INTRODUCCIÓN}

El uso de energía convencional deteriora al medio ambiente en que convivimos, por esta razón se hacen estudios en el uso de energías renovables, como es la energía solar térmica en sus diferentes aplicaciones; gracias a esto ha sido de gran interés, puesto que reduce las emisiones de $\mathrm{CO} 2$ y preserva el medio ambiente; creando iniciativas que promueven el uso de medios energéticos alternativos.

Actualmente existen empresas que se dedican a la fabricación de prototipos de refrigeración solar, entre las que sobresalen la empresa española Edibon, que se dedica a la fabricación y montaje de maqueta de refrigeración solar con todos los componentes del ciclo de refrigeración, controlada por una caja de interfaz a través de una PC, es un sistema TCRC que permiten la demostración del ciclo de refrigeración por compresión de vapor, este sistema es un equipo compacto de sobremesa que va montado en una estructura de aluminio anodizado y paneles en acero pintado.

Otra empresa que se destaca en el desarrollo de herramientas didácticas de laboratorio en el área de refrigeración y aire acondicionado es la empresa PA HILTON que desde 1959 desarrollan sistemas de refrigeración y aire acondicionados, reconocida por líderes del mercado con equipos para uso en las asignaturas universitarias de refrigeración, aire acondicionado y Transferencia de Calor. (P.A. Hilton Ltd, 2013)

En la FAREM - Estelí, ya existía este prototipo, haciendo falta su validación. En el año 2010 autoridades del SILAIS ESTELI donaron los componentes de este sistema, el profesor Edwin Reyes; responsable del laboratorio de Energías Renovables desinstaló un refrigerador solar en mal estado y luego con el profesor Luis Fuentes, diseñaron el prototipo que fue construido en la facultad pero este prototipo no se había sometido a evaluación paramétrica por estudiantes de la carrera de Ingeniería en Energías Renovables.

Por lo que el proceso de evaluación de este prototipo didáctico de refrigeración solar, tiene como interés principal de vincular la teoría recibida en el aula de clases con la práctica, puesto que consideramos que el aprendizaje así conseguido es más significativo y duradero en el tiempo.

En caso específico de nuestra facultad, egresados de la carrera de Ingeniería en Energías Renovables fueron participe de prácticas donde se aplicaron los conocimientos adquiridos en los planes de estudio de las asignaturas tales como termometría y termodinámica, turbomaquinas y climatización, disciplinas relacionadas con la refrigeración por expansión, por lo tanto el proceso de validación de un dispositivo funcional, nos conlleva alcanzar mejores niveles en proceso del aprendizaje, contribuyendo para una formación integral, promoviendo aplicaciones sencillas de leyes y principios para crear soluciones particulares frente a la necesidad y situaciones que conlleva este tipo de estudio.

\section{MATERIALES Y MÉTODOS}

\section{Ubicación del área de estudio}

El estudio se desarrolló en la Facultad Regional Multidisciplinaria de Estelí. FAREM-Estelí.

\section{Tipo de estudio}

Estudio cuantitativo y cualitativo de carácter concluyente que se realiza mediante encuestas y medición de variables de funcionamiento del refrigerador solar.

\section{El tipo de muestreo}

Muestreo probabilístico debido a que todos los individuos tienen la misma probabilidad de ser elegidos. 


\section{Fases de la investigación}

Para alcanzar los objetivos planteados, se ha utilizado el siguiente proceso metodológico:

\section{Etapa de campo}

\section{Evaluar los parámetros de funcionamiento del prototipo de refrigerador solar.}

Para poder determinar el funcionamiento del prototipo de refrigerador solar, lo cual es un punto fundamental en el proceso de validación de la investigación, se realizó la evaluación de los parámetros claves como radiación, temperatura y presión y se realizó de la siguiente manera:

Estando la refrigeradora en funcionamiento conectado a un panel solar de $175 \mathrm{w}$.

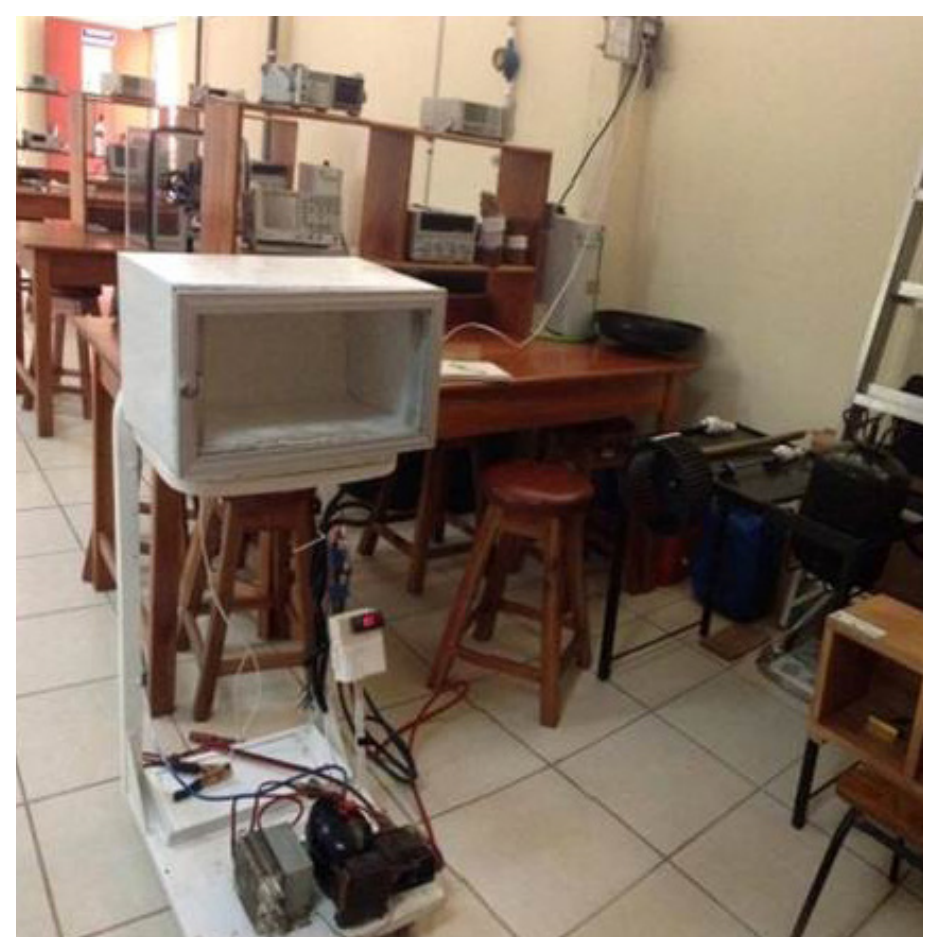

Imagen 1. Refrigerador solar conectado al panel solar

\section{Paso $\mathrm{N}^{\circ} 1$. Medición de la temperatura ${ }^{\circ} \mathrm{C}$}

Al iniciar el prototipo su funcionamiento, se efectuaron las mediciones de las temperaturas, el compresor arranca a temperatura ambiente y luego mediante se da el ciclo cerrado de refrigeración, comienza a bajar la temperatura la cual se va reflejando en el display del equipo refrigerador el cual registra los valores a través de un sensor conectado al freezer del mismo y este dato se comparó con un termopar colocado en el deposito congelador.

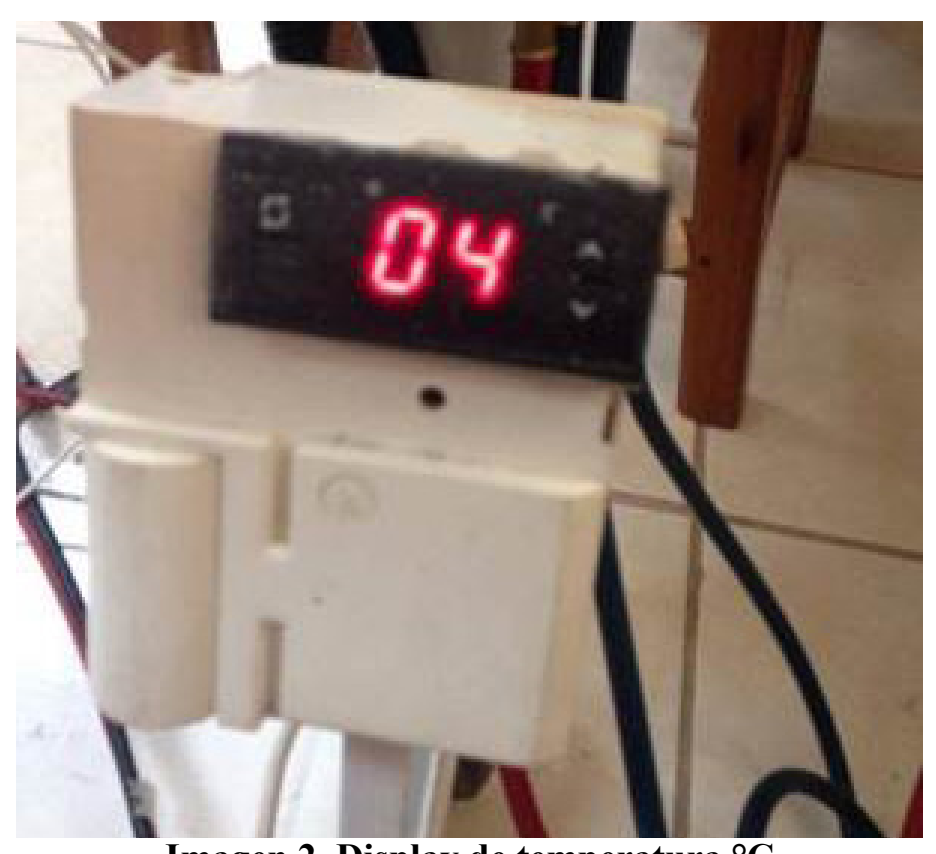

Imagen 2. Display de temperatura ${ }^{\circ} \mathrm{C}$

\section{Paso $N^{\circ} 2$. Medición de la Presión}

Para determinar los valores de presión en el funcionamiento del prototipo, se utilizó un juego de manómetros de presión. MANOMETRO DE CARATULA COLOR AZUL: Está diseñado para medir la presión en la línea succión o aspiración del compresor. En su escala, del cero hacia arriba nos mide presión por encima de la atmosférica y del cero hacia abajo nos mide presión de vacío en el sistema, es decir, presiones por debajo de la atmosférica. Su rango de medición es de cero a 250 PSIG y de cero hasta 29,92" de Hg. Igualmente dispone de sus equivalencias correspondientes en $\mathrm{Kg} / \mathrm{cm} 2 \mathrm{y} \mathrm{mm}$ de $\mathrm{Hg}$. 


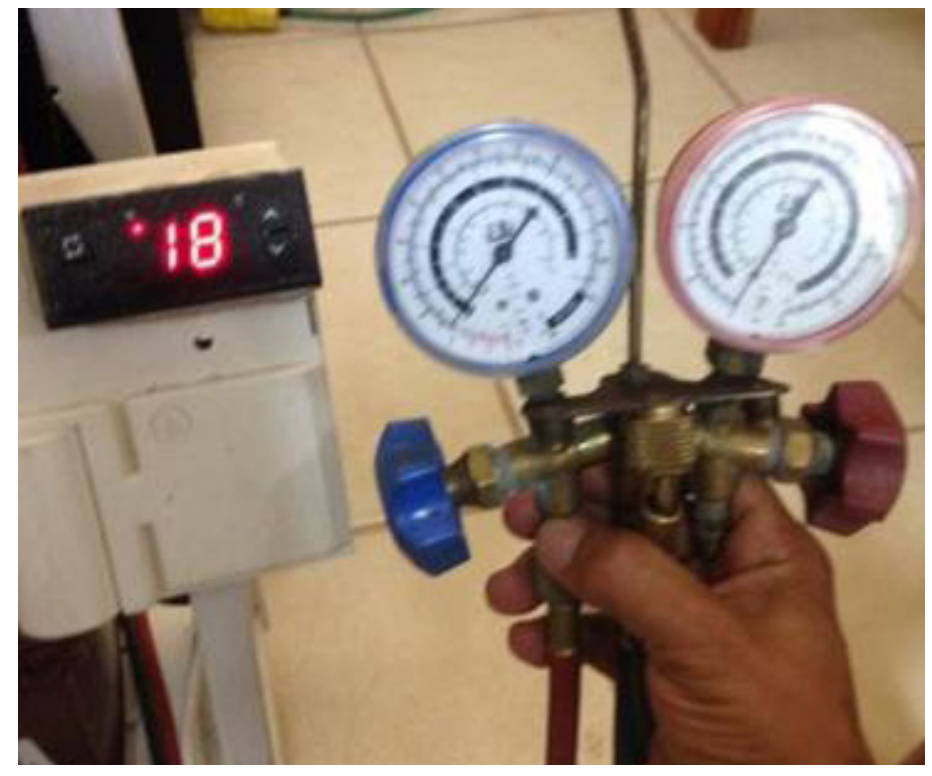

Imagen 3. Manómetro para medir la presión

\section{MANOMETRO DE CARATULA COLOR ROJO:}

Diseñado para medir las presiones de la línea descarga del compresor. Su rango es de cero hasta 500 PSIG (libras por pulgada cuadrada manómetricas). Igualmente pueden leerse las presiones en el sistema decimal de cero a $35 \mathrm{Kg} . / \mathrm{cm} 2$.

Cabe señalar, que para la medición de estos parámetros, se tomaron también los datos de radiación solar, para la comprobación de la hipótesis. Se utilizó un solarimetro cuya función es medir de manera muy precisa la radiación solar incidente sobre la superficie de la tierra.

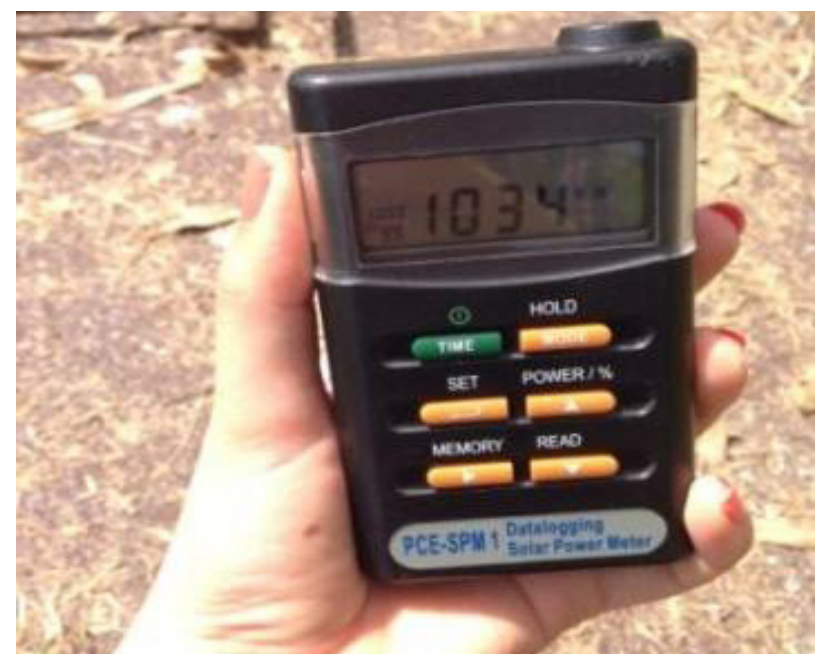

Imagen 4. Medición de la radiación solar con el solarímetro
Determinar la percepción de los estudiantes, de los diferentes años de la carrera de Ingeniería en Energías Renovables, sobre la importancia del prototipo de refrigeración solar en su formación académica.

La técnica utilizada para la recolección de información fue la encuesta cerrada. Esta se basó en un cuestionario, aplicado a una muestra de estudiantes de los diferentes años de la carrera de Ingeniería en Energías Renovables, a quienes se les plantearon preguntas con respecto a la percepción que ellos tienen sobre un prototipo de refrigeración solar, ya existente en nuestra facultad.

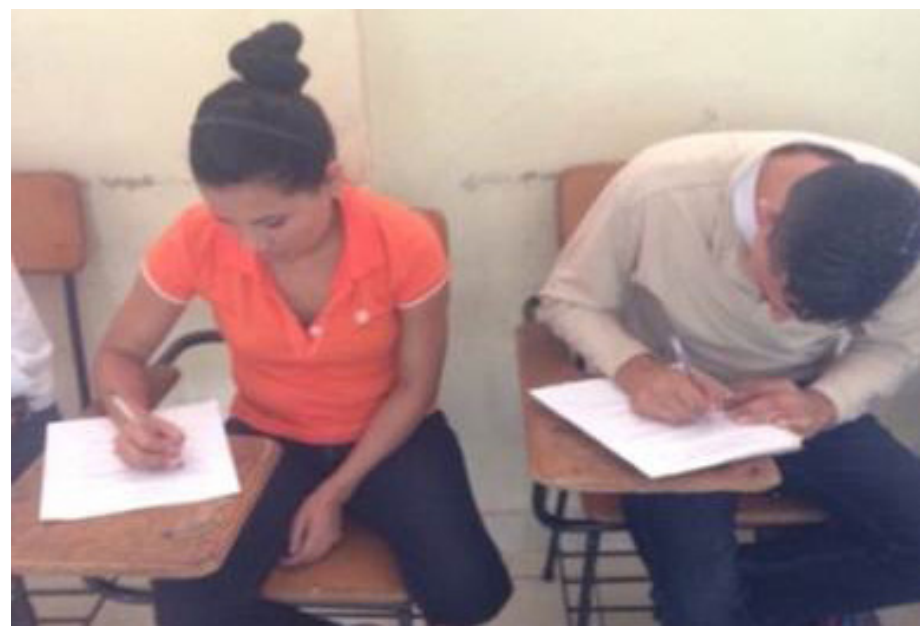

Imagen 4. Estudiantes contestando la encuesta

\section{RESULTADOS Y DISCUCIÓN}

En esta sección se abordara los resultados obtenidos acerca de la evaluación realizada al prototipo didáctico de refrigeración solar, así como la percepción de los estudiantes de Ingeniería en Energías Renovables del mismo.

Evaluar los parámetros de funcionamiento del prototipo de refrigerador solar.

\section{Temperatura}

Al conectarse al panel solar de $175 \mathrm{w}$ el refrigerador solar, inicio con una temperatura de $26^{\circ} \mathrm{C}$ ambiente, en ese instante las mediciones se tomaron cuando el dispositivo obtuvo una temperatura de $15^{\circ} \mathrm{C}$, hasta 
tener la temperatura más baja que fue de $4^{\circ} \mathrm{C}$. De esta manera nos damos cuenta, que la temperatura depende de la energía proporcionada por los paneles solares, debido a que su funcionamiento está enfocado a la energía solar. Así mismo cuando la radiación disminuía considerablemente, el compresor dejaba de funcionar y la temperatura en el display propio del equipo y la del datalogger de comparación empezaban su ascenso, es decir la temperatura aumentaba, sin embargo cuando conectábamos la batería de $12 \mathrm{v}, 100 \%$ cargada no se presentaba este problema, la temperatura iniciaba a ambiente y luego comenzaba a descender hasta la temperatura mínima de funcionamiento que en este caso es de $4^{\circ} \mathrm{C}$, el termostato de control cortaba a esta temperatura, ascendía a $6^{\circ} \mathrm{C}$ y después de tres minutos aproximadamente volvía a descender a $4^{\circ} \mathrm{C}$ y así sucesivamente, luego conectamos el sistema fotovoltaico completo: Panel, controlador, batería y refrigerador y realizamos medidas por un tiempo de aproximadamente tres días obteniendo los parámetros de evaluación.

\begin{tabular}{rrr}
\hline $\mathbf{T}^{\circ} \mathbf{C}$ & Presión PSI & Radiación Solar kw/m2 \\
\hline 15 & 20 & 1055 \\
14 & 10 & 1044 \\
11 & 5 & 1011 \\
6 & 3 & 998 \\
5 & 5 & 1015 \\
4 & 25 & 1016 \\
5 & 30 & 1080 \\
6 & 40 & 1047 \\
7 & 42 & 998 \\
6 & 3 & 700 \\
5 & 0 & 856 \\
4 & 25 & 789 \\
5 & 30 & 1115 \\
\hline
\end{tabular}

Tabla 1. Datos de evaluación del Refrigerador solar

\section{Presión}

Al mismo tiempo que mediamos temperatura se habían conectado los manómetros para las medidas de presión, el prototipo estando apagado tenía una presión de alta de
50 PSI, cuando se inició la toma de datos el refrigerador solar tenía una presión de 20 PSI, cuando este llego a la mínima temperatura registraba una presión de 25 PSI. Al igual que en la evaluación de la temperatura, la presión depende o varia con la radiación solar, debido a que al existir mejores condiciones de radiación solar toma al dispositivo llegar a la temperatura deseada en menor tiempo, lo que hace que el refrigerador solar sea más funcional y práctico.

Determinar la percepción de los estudiantes de los diferentes años de la carrera de Ingeniería en Energías Renovables sobre la importancia del prototipo de refrigeración solar.

En este acápite se abordan los resultados obtenidos por año, (I, II, III, IV) de la carrera de Ingeniería en Energías Renovables a través de la aplicación de la encuesta. (10 estudiantes por año obteniendo un total de 40 estudiantes.)

De acuerdo a las encuestas realizadas el 95\% de los estudiantes de la carrera de Ingeniería en Energías Renovables señalaron que es importante el uso de los paneles solares para la generación de energía, el resto señaló que no. Esto nos hace saber que para los estudiantes es importante el buen uso de la energía solar.

¿Considera importante el uso de la energía solar, por medio de paneles solares para la generación de energía?

$$
\square \mathrm{Si} \varpi \text { No }
$$

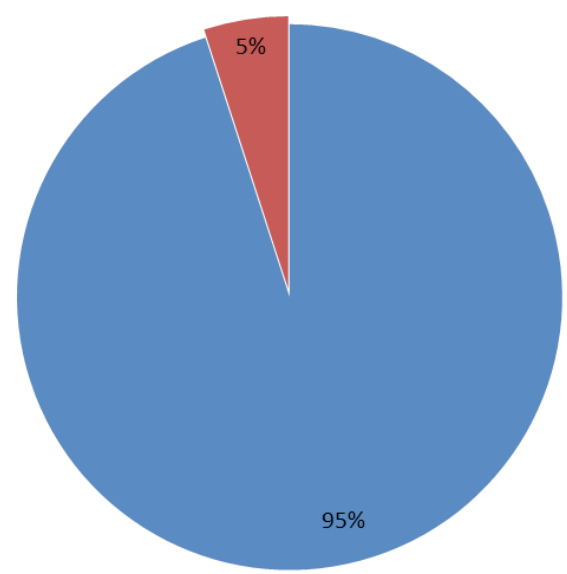


Con los datos conseguidos, se obtuvo que: el $97 \%$ de los encuestados, está de acuerdo que esta energía generada a través de los paneles solares, sea utilizada para el funcionamiento del refrigerador solar con fines didácticos, con estos resultados se observó la importancia de la inclusión de estos dispositivos para el aprovechamiento de la energía solar y sobre todo la vinculación de la teoría con la práctica.

¿Está de acuerdo que esa energía generada por medio de paneles solares, sea utilizada para el funcionamiento de un refrigerador solar con fines didácticos?

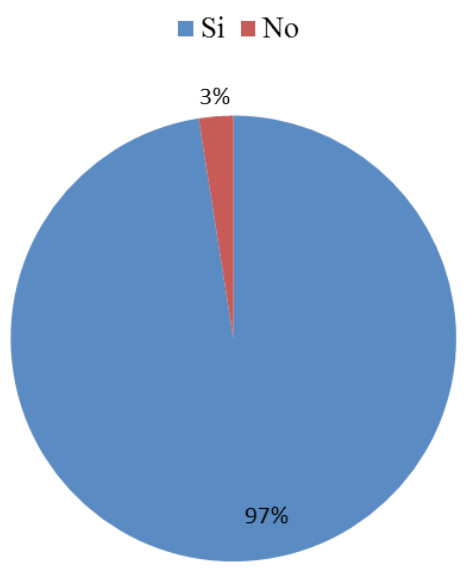

En la tercera pregunta de la encuesta aplicada a los estudiantes vemos que le $55 \%$ de ellos ha escuchado o tiene conocimiento de un sistema de refrigeración solar; mientas que el $45 \%$ respondió que no, con estos resultados se observa que es importante incrementar la inclusión de este prototipo en las carrera de Ingeniería en Energías Renovables, especialmente considerando en los primeros años de la carrera para aumentar el interés de los estudiantes, esto conlleva a vincular la teoría recibida en el aula de clase con la práctica; obteniendo un aprendizaje más significativo y duradero.
¿Ha escuachado o tiene conocimiento sobre un sistema de refrigeración solar?

$\square \mathrm{Si} \backsim$ No

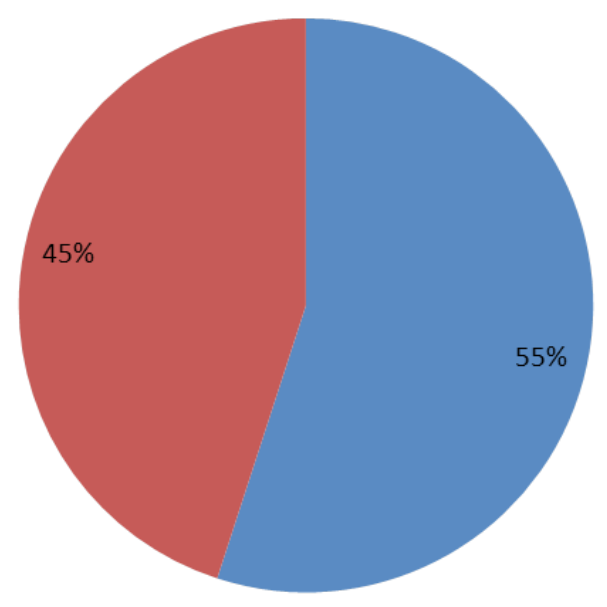

E1 92\% de los encuestados respondió que es relevante poder obtener más conocimientos sobre un sistema de refrigeración solar, $8 \%$ dijo que no. Estos resultados muestran que los estudiantes tienen una buena percepción sobre la inclusión de este prototipo con fines didácticos.

¿Cree relevante de acurdo a su área de estudio, poder obtener mas conocimientos, sobre un sistema de refrigeración solar?

$$
\square \mathrm{Si} \backsim \text { No }
$$

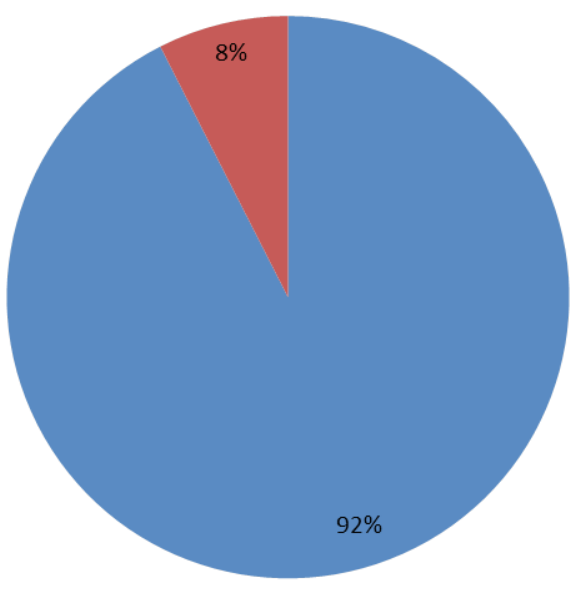

E1 92\% de los estudiantes señalaron que este dispositivo de refrigeración solar si es factible. Esto se debe al buen uso que como estudiantes le brindan a este prototipo para aumentar sus conocimientos. 
Factibilidad de contar con un prototipo en el laboratorio de energías renovables

$\because \mathrm{Si} \backsim \mathrm{No}$

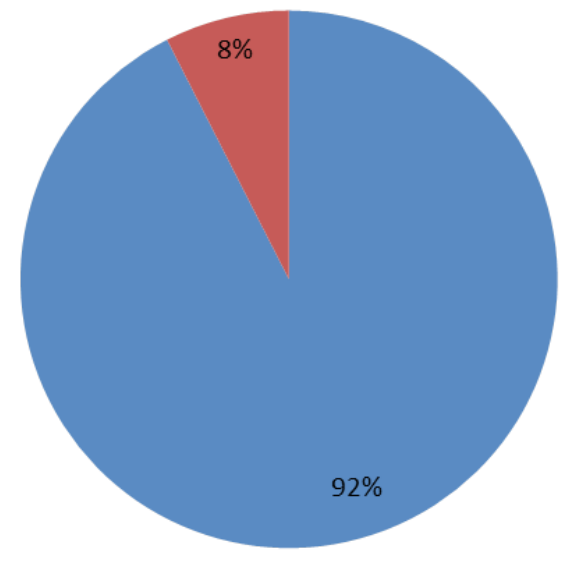

\section{CONCLUSIONES}

La temperatura y la presión en el refrigerador solar dependen de la radiación solar o del voltaje de la batería, debida ya que estas cambiaron rápidamente cuando la radiación era mayor o cuando el voltaje de la batería disminuía.

El aprovechamiento de la energía solar para el funcionamiento de un refrigerador solar, puede beneficiar los estudiantes tienen la oportunidad de realizar prácticas enfocadas a estos temas y de esta manera vincular la teoría recibida en el aula de clases con la práctica.

El uso de Energías Renovables para la creación de nuevas soluciones beneficia enormemente a la sociedad, sobre todo en aquellos lugares donde no hay acceso a la energía eléctrica, por lo tanto contar con un refrigerador solar mejora en estos casos el acceso a la salud.
Los estudiantes de la carrera de energías renovables ven necesario contar con un prototipo de esta naturaleza que les permita la obtención de conocimientos significativos de calidad.

La mayoría de los encuestados considera importante la inclusión de un prototipo didáctico de refrigeración solar con fines didácticos para así ampliar sus conocimientos.

\section{RECOMENDACIONES}

En caso de que se desee evaluar nuevamente el prototipo de refrigeración solar, se recomienda realizar las pruebas de temperatura y presión, en otra época del año, para obtener más radiación solar y así aumentar el rendimiento del dispositivo.

Promover el dispositivo en todos los años de Ingeniería en Energías Renovables.

Tomar una muestra más grande de encuestados por año.

\section{BIBLIOGRAFÍA DE REFERENCIA}

Gasquet H. (2006). Conversión de la energía solar fotovoltaica en energía eléctrica. México DF: Print . México.

Richard E Sontag. (1997). Termodinámica clásica y estadística. Michigan USA: Limusa.

Dyck Virz. (2008). Refrigeración comercial para técnicos de aire acondicionado. Madrid España: Paraninfo.

Edibon Internacional. (1 de Mayo de 2015). Equipamiento didáctico técnico. Recuperado el 2 de Junio de 2015, de http://www.edibon.com/ aboutus.php

Valadez, J. H. (2010). Manual de refrigeración doméstica. México DF: Trillas. 